\title{
Development of baroreflex control of heart rate in preterm and full term infants
}

\section{Gournay, E Drouin, J-C Rozé}

See end of article for

authors' affiliations

.....................

Correspondence to: Dr Gournay, Service de Cardiologie Pédiatrique, $\mathrm{CHU}$ de Nantes, BP 1005, 44035 Nantes Cedex,

France;

veronique.gournay@

chu-nantes.fr

Accepted

22 November 2001
Aim: To study baroreflex maturation by measuring, longitudinally, baroreflex sensitivity in preterm (gestational age 24-37 weeks) and full term infants.

Methods: Baroreflex sensitivity was quantified once a week, one to seven times, by a totally non-invasive method.

Results: Baroreflex sensitivity at birth was lower in the preterm infant and increased with gestational age. It also increased with postnatal age, but the values for the preterm infants at term still tended to be lower than the values for full term babies.

Conclusion: Baroreflex control of heart rate is present in the premature infant, but is underdeveloped and increases with postnatal age. Ex utero maturation seems to be delayed compared with in utero maturation assessed by full term values. These results may reflect sympathovagal imbalance in preterm infants and could identify a population more vulnerable to stress.
T he short term regulation of arterial blood pressure by the autonomic nervous system involves the arterial baroreflex. This reflex minimises any variation in blood pressure by responses of both heart rate and arterial vascular tone. For example, an increase in blood pressure will be buffered by a decrease in both heart rate and peripheral vascular resistance. These responses of heart rate and vascular resistance, produced by the efferent limb of the baroreflex, are mediated by the parasympathetic and sympathetic system respectively. As both systems are involved, studies on the baroreflex give information on sympathovagal balance.

Our knowledge about ontogeny of the baroreflex relies almost exclusively on animal studies, performed mainly in the lamb. Shinebourne et $a l^{1}$ have shown the presence of functional arterial baroreflex responses in sheep very early during fetal and postnatal life. Furthermore, these authors and others ${ }^{2}$ have shown that the arterial baroreflex is impaired in the fetus and newborn and increases later. Others have found, however, that the sensitivity of baroreflex control of the heart rate does not change throughout gestation ${ }^{3}$ or is higher during fetal life than postnatally. ${ }^{4}$ Few studies in humans have investigated the development of the baroreflex, possibly because the classical methods of investigation were not applicable to newborns for ethical reasons.

The classical methods of investigating baroreflex sensitivity (BRS) are based on analysis of changes in blood pressure and heart rate experimentally induced in a controlled fashion by various manoeuvres. In these methods, the change in blood pressure is produced by either pharmacological or mechanical means. The pharmacological methods consist of giving vasoactive drugs, such as phenylephrine and nitroprusside, whereas the mechanical methods consist of carotid baroreceptor deactivation or stimulation by body tilting or use of a neck chamber device. However, these methods have major limitations. Firstly, they are not very physiological and thus cannot be easily repeated many times over a short period. As a consequence, they cannot be used to provide information on the possible dynamic modulation of BRS over a 24 hour period, for example. Furthermore, results obtained by the pharmacological method may be marred by the possible influence of the drugs on the effectors of the baroreflex itself. Finally, it would seem unethical to use these methods to study the baroreflex in certain categories of human patients, such as infants, particularly sick infants.
All these potential limitations of the classical methods of measurement of BRS have led investigators to seek alternative methods. A non-invasive technique has been developed in humans and animals, ${ }^{5-7}$ in which recordings of blood pressure and electrocardiograms (ECGs) are analysed for the occurrence of sequences of spontaneously increasing or decreasing blood pressures and the beat to beat effect on the following cardiac intervals. Sequences of spontaneous variation compatible with a mechanism of baroreflex - that is, hypertension/ bradycardia and hypotension/tachycardia-are selected and analysed by linear regression of systolic blood pressure (SBP) versus the following pulse interval. The slope of this linear regression quantifies BRS. Thus analysis of spontaneously occurring variations in blood pressure and heart rate and, more specifically, assessment of their interactions may fruitfully be used to obtain information on baroreflex control of the heart. The non-invasive character of the spontaneous baroreflex method has been further improved by the availability of a reliable non-invasive device for continuous monitoring of blood pressure (Finapress 2300; Ohmeda, Englewood, Colorado, USA), rendering repeated measurements of BRS feasible even in human neonates. We have confirmed the reliability of blood pressure measurements obtained with this device in the newborn by comparing them with those obtained using an umbilical arterial catheter. ${ }^{8}$ Furthermore, we have reported preliminary data on non-invasive assessment of BRS in newborns.

The objective of our study was to quantify BRS noninvasively in premature and full term human newborns, and to study its maturation. To achieve this, we used spontaneous baroreflex measurement to obtain (a) repeated weekly measurements of BRS in a population of premature babies in the neonatal unit, from birth to discharge, and (b) one measurement in full term asymptomatic babies.

\section{METHODS}

\section{Subjects}

BRS data were collected in the neonatal intensive care unit of the University Hospital of Nantes. The ethics committee of the

Abbreviations: BRS, baroreflex sensitivity; SBP, systolic blood pressure 
hospital approved the study protocol. BRS was measured in 47 newborns who were in a stable respiratory and cardiac state. The study population was composed of two groups: 38 premature infants (gestational age 24-36 weeks) and nine full term infants. The latter were in the neonatal unit for various reasons, mainly suspicion of infection. However, they were all free from symptoms and were therefore considered to be representative of a healthy newborn population.

\section{Study design}

To determine the postnatal maturational pattern of baroreflex control of the heart rate, we repeated measurements in each premature infant at weekly intervals as many times as possible, depending on the length of their stay and their haemodynamic and respiratory state.

Full term newborns were tested only once because of their early discharge home (their mean duration of stay was less than 3 days).

The studies were performed between 0800 and 1400 hours in babies who were peacefully awake or sleeping. Sleep state was not determined.

\section{Methods of measurement}

Our method of non-invasive measurement of BRS has been described previously. ${ }^{9}$ Briefly, heart rate and arterial blood pressure were recorded continuously. Heart rate was monitored with an ECG monitor (Mingograph 34; Siemens, Erlangen, Germany). Arterial blood pressure was monitored by the volume clamp method using a Finapress 2300. ECG and arterial blood pressure were converted from analogue to digital format with a temporal resolution of $200 \mathrm{~Hz} /$ channel and an amplitude resolution of 12 bits. The converted signals were stored in a computer (486 SX). All data acquisitions and analyses were performed with a menu driven software package.

Baseline values were recorded for ten minutes, and a BRS value was then obtained from four consecutive four minute periods of recording, each separated by ten minutes (the whole study therefore lasted for about one hour). In each four minute period, we selected a very short sequence (lasting a few seconds) of at least ten cardiac cycles, during which SBP varied rapidly over a few beats by $10-20 \%$ from baseline, inducing an immediate heart rate response consistent with baroreflex activity - that is, either bradycardia in response to an increase in SBP $(+\Delta R R /+S B P)$, or tachycardia in response to a decrease in SBP $(-\Delta R R /-S B P)$, where $R R$ is pulse interval. The BRS value was measured from the data recorded during these four short sequences. For each sequence, a single value of BRS was provided by the slope of the regression line between SBP and the following pulse interval. The slope of the regression line and the corresponding correlation coefficient $(r)$ were calculated. We considered the data suitable for BRS measurement only if at least three measurements out of four produced consistent results-that is, the difference between two values did not exceed $15 \%$ of the mean of the two values-and if the regression line was composed of a minimum of 10 points - that is, 10 cardiac cycles - with a correlation coefficient $r>0.80$. In each study, the mean value of the three or four accepted slopes was considered as the index of BRS.

\section{Data analysis}

Results are expressed as mean (SE). For statistical purposes, the values were classified into three groups of postnatal age (1-10, 11-30, > 30 days) in the longitudinal study. If, in a single patient, multiple points were obtained for one given postnatal age group because of weekly repeated measurements, these points were averaged to provide one value per group. Data were compared in the longitudinal study between different groups of postnatal age by analysis of variance for repeated

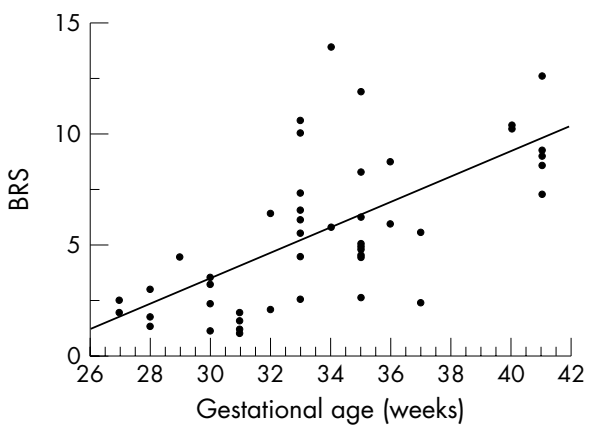

Figure 1 Increase in baroreflex sensitivity (BRS) with gestational age $\left(p<0.0001 ; r^{2}=0.438 ; B R S=-14.012+0.577 G A\right.$ where $\mathrm{GA}$ is gestational age).

measurements. Post hoc analyses were performed with a Bonferroni/Dunn test. The relation between gestational age and BRS was studied by multiple regression, with the first BRS value measured in each patient as the dependent variable, and gestational age and postnatal age as independent variables. Postnatal age was included in this multiple regression to eliminate a possible effect of postnatal age on the effect of gestational age, as the postnatal age at which the first BRS value was measured varied among the patients. Differences were considered significant when $\mathrm{p}<0.05$.

\section{RESULTS}

We obtained 141 BRS values from 47 newborns. The mean gestation of the premature infants was $30.6(0.4)$ weeks. The mean birth weight of the 38 premature infants was 1620 (75) $\mathrm{g}$, and for the nine full term infants it was 3050 (260) g. Only one measurement was taken in a patient who was intubated and mechanically ventilated, six were taken in infants on continuous positive airway pressure, 15 in those on nasal oxygen, and the remaining 119 were taken in infants breathing room air. Twenty seven BRS values were obtained from infants receiving methylxanthenes.

Initial BRS values increased significantly with gestational age $(\mathrm{BRS}=-14.012+0.577 \mathrm{GA}$ where GA is gestational age; $r^{2}=0.438 ; \mathrm{p}<0.0001$ ), independently of postnatal age (fig l).

\section{Longitudinal study}

To determine the postnatal maturational pattern of baroreflex control of heart rate, we repeated measurements in each premature infant at weekly intervals as many times as possible depending on the length of stay. The number of BRS values per infant ranged from one to seven in the most premature infants. However, there were missing data in several cases because the infant either could not be tested (because of haemodynamic instability, for example) or was transferred to another neonatal centre closer to home. Finally, we were able to obtain enough weekly measurements to perform a true longitudinal study in a subgroup of eight premature infants under 32 weeks of gestational age - that is, we obtained at least one point in each of the three categories of postnatal age: 1-10 days, $11-30$ days, $>30$ days.

There was significant postnatal maturation in the subgroup of eight very premature infants studied longitudinally, from $1.8(0.139) \mathrm{ms} / \mathrm{mm} \mathrm{Hg}$ before 10 days of age, to $2.37(0.23)$ $\mathrm{ms} / \mathrm{mm} \mathrm{Hg}$ between 11 and 30 days of age, to 3.47 (0.471) $\mathrm{ms} / \mathrm{mm} \mathrm{Hg}$ after 30 days of age $(\mathrm{p}=0.0051)$ (fig 2 ).

To determine whether ex utero maturation differs from in utero maturation, we compared BRS values of very premature infants measured when they reached term with BRS values of full term infants. The value when the most premature infants (born before 33 weeks) reached term (5.61 (1.37) ms/mm Hg; $\mathrm{n}=5$ ) was lower than that of full term infants (8.94 (0.92) 


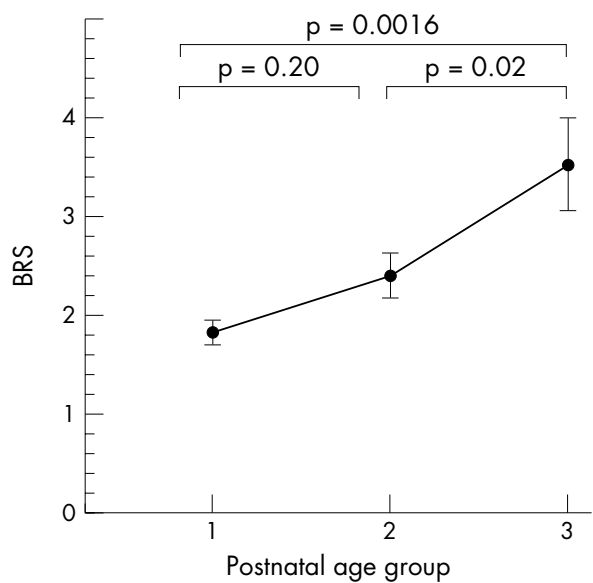

Figure 2 Increase in baroreflex sensitivity (BRS) with postnatal age in a subgroup of eight very premature infants studied longitudinally. Postnatal age groups: 1, 1-10 days; $2,11-30$ days; $3,>30$ days) Error bars indicate SEs.

$\mathrm{ms} / \mathrm{mm} \mathrm{Hg} ; \mathrm{n}=9)(\mathrm{p}=0.06)$, suggesting that BRS maturation may be delayed in very preterm infants.

\section{DISCUSSION}

In this study, we showed that BRS quantified non-invasively in human newborns increases with gestational age. Furthermore, although BRS increases with postnatal age in premature infants, it is still depressed when they reach term compared with full term newborns.

The method we used to quantify BRS has the advantage of being totally non-invasive. We have previously confirmed its feasibility and reproducibility. ${ }^{9}$ The BRS values measured during hypotension/tachycardia sequences do not differ significantly from those measured during hypertension/bradycardia sequences in the premature or full term infants. ${ }^{9}$ However, it is relatively new and some may claim that it is not as reliable as the classical invasive methods. Convincing evidence that this non-invasive technique provides reliable information on the sensitivity of the arterial baroreflex has been published. Firstly, results obtained using this method are profoundly altered by baroreceptor denervation. The almost complete disappearance of hypertension/bradycardia and hypotension/ tachycardia sequences in conscious cats after sinoaortic denervation strongly supports the involvement of arterial baroreflexes in the genesis of these phenomena. ${ }^{5}$ A second line of evidence supporting the reliability of non-invasive quantification of BRS is provided by its good correlation with pharmacological measurements in animals and humans. ${ }^{10} 11$ Finally, it is noteworthy that a method similar to ours has been used by other investigators to study BRS in newborns. ${ }^{12}$ The objective of that study was to quantify BRS from prolonged continuous recordings of blood pressure and heart rate in newborns of 35-42 weeks gestation during and after extracorporeal membrane oxygenation. BRS measurements were derived from segments of spontaneous variations of blood pressure with simultaneous changes in heart rate consistent with the presence of a baroreflex. In these near term critically ill newborns, Buckner et al showed a functional baroreflex capable of resetting.

Our results agree with those from a study of possible maturational changes in BRS with advancing gestational age and postnatal age in the human neonate. ${ }^{13}$ As in our study, infants were tested in a longitudinal fashion once a week beginning at 28-32 weeks of gestational age, by analysing heart rate variability by spectral analysis in the supine position and after head up tilting. Like ours, these findings suggested that the cardiac baroreflex is poorly developed in premature infants, and that it becomes more functional with postnatal development. In the few other studies available on the ontogeny of BRS in the human newborn, the baroreflex was evaluated by the magnitude of the heart rate response to body tilting. With this method, results were controversial: some $^{14}$ reported a poorly developed response in the newborn, independent of the length of gestation, age after birth, and respiratory status, whereas others ${ }^{15-17}$ observed a well developed response in healthy premature and full term infants. Results from animal studies are even more conflicting. Indeed, Shinebourne et al ${ }^{1}$ reported that BRS was greater in the newborn lamb than in the fetus. However, subsequent studies in the lamb by Dawes et $a l^{2}$ and Maloney et $a l^{3}$ failed to support these observations. Segar et $\mathrm{al}^{4}$ even reported greater BRS in fetuses than in newborn and 4-6 week old lambs. Similarly, investigators disagree about the way in which BRS varies in pigs with postnatal maturation: Palmisano et $a l^{18}$ found that BRS decreased with increasing age in the piglet, whereas Patton et a $^{19}$ reported an increasing BRS from birth to 6 weeks of age.

We can only speculate on the possible mechanisms underlying the developmental changes in BRS that we observed. Arterial baroreceptor reflex control of heart rate involves three main components: the afferent limb consisting of the baroreceptors, the nucleus of the solitary tract in the medulla, and the efferent limb consisting of the cardioinhibitory vagal innervation. The hypothesis of underdeveloped afferent baroreceptor activity to account for the lower BRS values observed in premature newborns appears unlikely. Indeed, the study of Blanco et $a l^{20}$ in the fetal lamb showed functional baroreceptors, the sensitivity of which decreased over the last third of gestation. Thus the depressed BRS in premature infants is more likely to be due to impairment of the central integration of baroreceptor input and/or the efferent limb of the reflex, namely the vagal innervation of the heart. The increase in BRS with maturation probably parallels the development of sympathovagal balance, with a progressive increase in reflex vagal activity.

This increase in reflex vagal activity can be paralleled to a progressive increase in vagal tone which has been shown by non-invasive studies in premature and full term infants, using power spectral analysis of heart rate variability, another method of assessing the autonomic nervous system. The high frequency component of heart rate variability, centred around respiratory frequency, is considered to reflect parasympathetic activity, whereas mid and low frequency components are of mixed sympathetic and parasympathetic origin. Using this method, two studies have shown a progressive decrease in heart rate and increase in high frequency variability with gestational and conceptional age, indicating an increase in tonic vagal activity. ${ }^{21}{ }^{22}$ Likewise, several authors reported a diminished vagal component of heart rate control in prematures reaching term, as compared with full term newborns, ${ }^{23}{ }^{24}$ which can be paralleled to our observation of delayed maturation of BRS in prematurely born infants reaching term. Interestingly, according to Porges, ${ }^{24}$ this depressed vagal tone in prematures suggests greater vulnerability to stress and may identify a "high risk" population. This view is supported by studies showing that sick preterm infants have higher heart rates and poor heart rate variability, suggesting depressed vagal tone, compared with healthy infants at comparable gestational ages. ${ }^{25}{ }^{26}$ Furthermore, the reappearance of normal heart rate variability in these sick infants is associated with a good prognosis.

To enhance our understanding of the mechanisms of heart rate control in the neonate by the autonomic nervous system, it would be of interest to measure both BRS and heart rate variability. Indeed, when these two variables were evaluated and compared in a group of adult patients with a prior myocardial infarction, ${ }^{27}$ although BRS was significantly $(\mathrm{p}<0.01)$ correlated with the various measures of heart rate variability, this correlation was weak $(r=0.57-0.63)$. This 
finding implies that the two methods explore different aspects of the autonomic control of heart rate. Heart rate variability primarily reflects tonic vagal activity, whereas BRS measures predominantly reflex vagal activity.

BRS in healthy newborns may be modulated by other factors that we did not look at. For example, we will have to determine in further studies whether sleep state, which is known to modulate vagal activity, significantly influences BRS.

The point of assessing BRS is to provide information about maturation of the autonomic nervous system, which is important in numerous situations in the clinical setting. For example, the apnoea/bradycardia syndrome of the premature infant reaching term may involve long lasting effects of premature birth on central and peripheral mechanisms that control heart rate. ${ }^{26}$ Other examples are provided by pathologies in which brain stem dysfunction is suspected, such as fetal asphyxia or congenital malformations. Finally, it would be interesting to study BRS maturation in a population of infants with apparent life threatening events, at the origin of which sympathovagal imbalance may be involved.

In conclusion, BRS quantified non-invasively in human newborns increases with gestational and postnatal age. Furthermore, this increase with maturation, although observed from birth to term in premature infants, seems to be delayed compared with that of full term newborns.

\section{ACKNOWLEDGEMENTS}

This work was supported in part by a governmental grant $65 / 6-\mathrm{H}$ (Projet Hospitalier de Recherche Clinique 1995) from the Ministère de la Santé. We also thank Ohmeda (France) for providing the Finapress 2300.

\section{Authors' affiliations}

V Gournay, Service de Cardiologie Pédiatrique, CHU de Nantes, BP 1005, 44035 Nantes Cedex, France

E Drouin, J-C Rozé, Service de Réanimation Néonatale et Pédiatrique, $\mathrm{CHU}$ de Nantes

\section{REFERENCES}

1 Shinebourne EA, Vapaavuori RL, Williams RL, et al. Development of baroreflex activity in unanesthetized fetal and neonatal lambs. Circ Res 1972;31:710-18.

2 Dawes GS, Johnston BM, Walker DW. Relationship of arterial pressure and heart rate in fetal, newborn and adult sheep. J Physiol (Lond) 1980;309:405-17.

3 Maloney JE, Cannata JP, Dowling MH, et al. Baroreflex activity in conscious fetal and newborn lambs. Biol Neonate 1977;31:340-50.
4 Segar JL, Hajduczok G, Smith BA, et al. Ontogeny of baroreflex control of renal sympathetic nerve activity and heart rate. Am J Physiol 1992;263:H1819-26.

5 Bertinieri G, DiRienzo M, Cavallazzi A, et al. A new approach to analysis of the arterial baroreflex. J Hypertens 1985;3(suppl 3):s79-81.

6 Bertinieri G, DiRienzo M, Cavallazzi A, et al. Evaluation of baroreceptor reflex by blood pressure monitoring in unanesthetized cats. Am J Physiol 1988;254:H377-83.

7 Parati G, DiRienzo M, Bertinieri G, et al. Evaluation of the baroreceptor-heart rate reflex by 24 -hour intra-arterial blood pressure monitoring in humans. Hypertension 1998;12:214-22.

8 Drouin E, Gournay V, Calamel J, et al. Feasibility of using finger arterial pressure in neonates. Arch Dis Child Fetal Neonatal Ed 1997;77:F139-40.

9 Drouin E, Gournay V, Calamel J, et al. Assessment of spontaneous baroreflex sensitivity in neonates. Arch Dis Child Fetal Neonatal Ed 1997;76:F108-12.

10 Frankel RA, Metting PJ, Britton SL. Evaluation of spontaneous baroreflex sensitivity in conscious dogs. J Physiol (Lond) 1993;462:31-45.

11 Parlow J, Viale JP, Annat G, et al. Spontaneous cardiac baroreflex in humans. Comparison with drug-induced responses. Hypertension 1995;25:1058-68.

12 Buckner PS, Maidens JM, Finer NN. Characterization of the neonatal heart rate baroreflex during and after ECMO. Early Hum Dev 1993;32:49-61.

13 Mazursky JE, Birkett CL, Bedell KA, et al. Development of baroreflex influences on heart rate variability in preterm infants. Early Hum Dev 1998;53:37-52.

14 Holden K, Morgan JS, Krauss AN, et al. Incomplete baroreceptor responses in newborn infants. Am J Perinatol 1985;2:31-4.

15 Finley JP, Hamilton R, MacKenzie MG. Heart rate response to tilting in newborns in quiet and active sleep. Biol Neonate 1984;45:1-10.

16 Chen CM, Tsai TC, Lan MC. Effect of body tilting on physiologic functions in healthy term neonates. Acta Paediatr 1995:84:474-7.

17 Thoresen M, Cowan F, Walloe L. Cardiovascular responses to tilting in healthy newborn babies. Early Hum Dev 1991;26:213-22.

18 Palmisano BW, Clifford PS, Coon RL, et al. Development of baroreflex control of heart rate in swine. Pediatr Res 1989;27:148-52.

19 Patton DJ, Hanna BD. Postnatal maturation of baroreflex heart rate control in neonatal swine. Can J Cardiol 1994;10:233-8.

20 Blanco CE, Dawes GS, Hanson MA, et al. Carotid baroreceptors in fetal and newborn sheep. Pediatr Res 1988;24:342-6

21 Chatow U, Davidson S, Reichman BL, et al. Development and maturation of the autonomic nervous system in premature and full-term infants using spectral analysis of heart rate fluctuations. Pediatr Res 1995; 37:294-302.

22 Clairambault J, Curzi-Dascalova L, Kauffman F, et al. Heart rate variability in normal sleeping full-term and preterm neonates. Early Hum Dev 1992;28: 169-83.

23 Eiselt M, Curzi-Dascalova L, Clairambault J, et al. Heart rate variability in low-risk prematurely born infants reaching normal term: a comparison with full-term newborns. Early Hum Dev 1993;32:183-95.

24 Porges SW. Vagal tone: a physiologic marker of stress vulnerability. Pediatrics 1992;90:498-504.

25 Cabal LA, Siassi B, Zanini B, et al. Factors affecting heart rate variability in preterm infants. Pediatrics 1980;65:50-6

26 Henslee JA, Schechtman VL, Lee MY, et al. Developmental patterns of heart rate and variability in prematurely-born infants with apnea of prematurity. Early Hum Dev 1997;47:35-50.

27 Bigger JT Jr, La Rovere MT, Steinman RC, et al. Comparison of baroreflex sensitivity and heart period variability after myocardial infarction. J Am Coll Cardiol 1989;14:1511-18. 